\title{
DISPERSIVE RAREFACTION WAVE WITH A LARGE INITIAL GRADIENT ${ }^{1}$
}

\author{
Alexander E. Elbert \\ Krasovskii Institute of Mathematics and Mechanics \\ of Ural Branch of Russian Academy of Sciences, Ekaterinburg, Russia \\ E-mail: aee@imm.uran.ru \\ Sergey V. Zakharov \\ Krasovskii Institute of Mathematics and Mechanics \\ of Ural Branch of Russian Academy of Sciences, Ekaterinburg, Russia \\ E-mail: svz@imm.uran.ru
}

\begin{abstract}
Consider the Cauchy problem for the Korteweg-de Vries equation with a small parameter at the highest derivative and a large gradient of the initial function. Numerical and analytical methods show that the obtained using renormalization formal asymptotics, corresponding to rarefaction waves, is an asymptotic solution of the KdV equation. The graphs of the asymptotic solutions are represented, including the case of non-monotonic initial data.
\end{abstract}

Key words: The Korteweg-de Vries, Cauchy problem, Asymptotic behavior, Rarefaction wave.

\section{Introduction}

Consider the Cauchy problem for the Korteweg-de Vries equation:

$$
\begin{gathered}
\frac{\partial u}{\partial t}+u \frac{\partial u}{\partial x}+\varepsilon \frac{\partial^{3} u}{\partial x^{3}}=0, \quad t \geqslant 0, \quad \varepsilon>0, \\
u(x, 0, \varepsilon, \rho)=\Lambda\left(\frac{x}{\rho}\right), \quad x \in \mathbb{R}, \quad \rho>0,
\end{gathered}
$$

with a bounded initial function $\Lambda$, which has finite limits $\Lambda_{0}^{ \pm}=\lim _{s \rightarrow \pm \infty} \Lambda(s), \Lambda_{0}^{-}<\Lambda_{0}^{+}$, and its derivative quickly enough tends to zero at infinity. This is a classic model of nonlinear waves in the medium with small dispersion. For the case of discontinuous initial functions the asymptotics was studied by A.B. Gurevich and L.P. Pitaevskii [1]. The asymptotic formulas for the evolution of rarefaction waves were also found in the works $[2,3]$ by the Whitham method and in the work [4] by the inverse scattering method. For the initial step-like function the asymptotic formulas are obtained by the method of the inverse scattering [5-7]. In the case of a smoothed step-like initial function the asymptotic expansion was constructed by the method of matching in the work [8]. We emphasize that the study of analytic properties of solutions of the KdV equation and their asymptotic behavior is one of the interesting problems of the modern mathematical physics [9],[17][26]. It should be noted that the KdV equation remains a most important model in hydrodynamics [31] and physics of plasma up to subnuclear scales [29]. In particular, the latest research was aimed to the study of rarefaction waves $[34,35]$.

\footnotetext{
${ }^{1}$ This research was supported by RFBR grant No. 14-01-00322.
} 
We assume that a smooth initial function $\Lambda: \mathbb{R} \rightarrow \mathbb{R}$ has finite limits $\Lambda_{0}^{ \pm}=\lim _{\sigma \rightarrow \pm \infty} \Lambda(\sigma)$, and the asymptotic expansions satisfy the asymptotic formula

$$
\Lambda(\sigma)=\sum_{n=0}^{\infty} \frac{\Lambda_{n}^{ \pm}}{\sigma^{n}}, \quad \sigma \rightarrow \pm \infty
$$

The existence of a classical smooth solution of problem (1.1)-(1.2) is guaranteed by the Kappeler theorem [10] if

$$
\int_{-\infty}^{0}\left|\Lambda(x)-\Lambda_{0}^{-}\right|\left(1-x^{3}\right) d x<\infty, \quad \int_{0}^{\infty}\left|\Lambda(x)-\Lambda_{0}^{+}\right|\left(1+x^{3}\right) d x<\infty .
$$

Here, however, it is possible not to assume these restrictions, but we may consider only the formal asymptotic solution; moreover, in a special class of functions the existence of solutions is proved for unbounded initial data [11].

In this article the approximation constructed in the work [12] is refined, and it is shown in Section 3 that it is the asymptotic solution for the problem (1.1)-(1.2) as $\varepsilon \rightarrow 0, \rho \rightarrow 0$ and the ratio of the parameters $\rho^{2} / \varepsilon \rightarrow 0$, and also numerical analysis has been performed for this solution in Section 4. These results show the efficiency of the renormalization approach used in paper [12]. One of the purposes of the paper is to understand and determine the precise mathematical meaning of "formal asymptotic solutions" of the KdV equation. Since for a similar problem for a parabolic equation the closeness of the asymptotics obtained by renormalization, to the exact solution, was proved [16], so there is some reason to suggest that for the KdV equation the formal asymptotic solution, which is found by the same method, also approximate the exact solution.

It is clear that the structure of the asymptotics must essentially depend on the ratio of the parameters $\varepsilon$ and $\rho$. Here we assume the following conditions:

$$
\mu=\frac{\rho}{\sqrt{\varepsilon}} \rightarrow 0
$$

A similar problem for compression waves was studied in the work [33]. In the present paper, we have to use another definition of the asymptotic solution (different from [33]) because of the specificity of its behavior.

\section{Renormalization}

It is known that in some cases the behavior of the solutions of singularly perturbed differential equations with a small parameter at the highest derivative becomes in some sense self-similar. Then the analysis of solutions using the method of renorm-group [13] becomes effective. This approach has the advantage that we immediately get the uniform approximation of the problem, which eliminates the need to build asymptotic ansatzes in different areas. For example, the composite asymptotic solution of the Cauchy problem with the condition (1.2) was obtained by matching [14] for a quasilinear parabolic equation [15], and in [16] it is shown that the renormalization approximation of the solution is asymptotically close to the composite asymptotic solution.

The relationship between self-similarity of the solutions and the parameter $\mu=\frac{\rho}{\sqrt{\varepsilon}}$ was shown already in the work of Yu. A. Berezin and V. Karpman [28] in connection with the study of the evolution of perturbations in plasma. 
We will construct asymptotic solution of the problem (1.1)-(1.2), using a technique similar to the method of renormalization group, in the most simple version. Let us introduce the inner variables

$$
x=\sqrt{\varepsilon} \eta, \quad t=\sqrt{\varepsilon} \theta,
$$

since this takes into account all terms of equation (1.1). We take the solution of the equation

$$
\frac{\partial Z}{\partial \theta}+Z \frac{\partial Z}{\partial \eta}+\frac{\partial^{3} Z}{\partial \eta^{3}}=0
$$

with the initial condition

$$
Z(\eta, 0)= \begin{cases}\Lambda_{0}^{-}, & \eta<0 \\ \Lambda_{0}^{+}, & \eta>0\end{cases}
$$

as a "starting" function. We seek the expansion of the solution in the following form

$$
u(x, t, \varepsilon, \rho)=Z(\eta, \theta)+\mu W(\eta, \theta, \mu)+\ldots,
$$

where the additive $\mu W(\eta, \theta, \mu)$ is supposed to eliminate the "starting" function singularity at the initial time. From equations (1.1) and (2.2) it follows that the function $W$ satisfies the linear equation

$$
\frac{\partial W}{\partial \theta}+\frac{\partial(Z W)}{\partial \eta}+\frac{\partial^{3} u}{\partial \eta^{3}}=0 .
$$

Differentiating the equation (2.2) with respect to the variable $\eta$, we see that the expression

$$
G(\eta, \theta)=\frac{1}{\Lambda_{0}^{+}-\Lambda_{0}^{-}} \frac{\partial Z(\eta, \theta)}{\partial \eta}
$$

satisfies the equation (2.5). Moreover, $G$ is the Green function, since

$$
\lim _{\theta \rightarrow+0} \int_{-\infty}^{\infty} G(\eta, \theta) f(\eta) d \eta=-\frac{1}{\Lambda_{0}^{+}-\Lambda_{0}^{-}} \int_{-\infty}^{\infty} Z(\eta, 0) f^{\prime}(\eta) d \eta=f(0)
$$

for any finite functions $f$.

We will find a solution $W$ in the form of a convolution with the Green function $G$ such that the asymptotic approximation will satisfy the initial condition (1.2). Then

$$
W=\frac{1}{\Lambda_{0}^{+}-\Lambda_{0}^{-}} \int_{-\infty}^{\infty} \frac{\partial Z(\eta-\mu s, \theta)}{\partial \eta}[\Lambda(s)-Z(s, 0)] d s .
$$

After integration by parts and substitution of the expansion (2.4) we get the desired expression

$$
u \approx \frac{1}{\Lambda_{0}^{+}-\Lambda_{0}^{-}} \int_{-\infty}^{\infty} Z\left(\frac{x-\rho s}{\sqrt{\varepsilon}}, \frac{t}{\sqrt{\varepsilon}}\right) \Lambda^{\prime}(s) d s,
$$

which clarifies the structure of the asymptotic solution in the parameters $\varepsilon$ and $\rho$ in the leading approximation. Despite the fact that the functions $Z$ and $W$ do not depend explicitly on $\sqrt{\varepsilon}$, the result depends on $\mu$ and $\sqrt{\varepsilon}$ because of the change (2.1) since the asymptotic solution (2.6) is also considered at finite values of time $t$.

These calculations are given here for the convenience of the reader and a logical passage to the next section, where we use the result of [12] based on formula (2.6) to obtain a more suitable expression for numerical computations. 


\section{Formal asymptotic solution}

We seek the asymptotic solution $\tilde{u}$ in the form

$$
\begin{aligned}
& \tilde{u}(x, t)=R\left(\sigma, \mu^{2} \omega\right)+S(\sigma, \omega), \\
& \sigma=\frac{x}{\rho}, \quad \omega=\frac{\varepsilon t}{\rho^{3}}, \quad \mu=\frac{\rho}{\sqrt{\varepsilon}},
\end{aligned}
$$

where the function

$$
R\left(\sigma, \frac{t}{\rho}\right)=\int_{0}^{1} \Lambda\left(\sigma+\frac{c t y}{\rho}\right) d y
$$

according to [12], is obtained by substitution of the asymptotics of the Gurevich-Pitaevskii solution [1] for the rarefaction wave for $\Lambda_{0}^{+}=0$ and $\Lambda_{0}^{-}=-\frac{c}{6}(c>0)$ in the formula $(2.6)$.

The function $S$ satisfies the equation

$$
\frac{\partial S}{\partial \omega}+\frac{\partial^{3} S}{\partial \sigma^{3}}=-\frac{\partial^{3} R}{\partial \sigma^{3}}, \quad S(\sigma, 0)=0
$$

to compensate the leading term with $\frac{\partial^{3} R}{\partial x^{3}}$ in the $\mathrm{KdV}$ equation (1.1). The solution $S$ can be written in the following form:

$$
S=-\int_{0}^{\omega} \frac{1}{\sqrt[3]{3\left(\omega-\omega^{\prime}\right)}} \int_{-\infty}^{\infty} \operatorname{Ai}\left(\frac{\sigma-\sigma^{\prime}}{\sqrt[3]{3\left(\omega-\omega^{\prime}\right)}}\right) \frac{\partial^{3} R\left(\sigma^{\prime}, \mu^{2} \omega^{\prime}\right)}{\partial \sigma^{\prime 3}} d \sigma^{\prime} d \omega^{\prime} .
$$

Proposition 1. Let $\mu<1, \Lambda \in C^{6}(\mathbb{R})$,

$$
\lim _{s \rightarrow-\infty} \Lambda(s)=-\frac{c}{6}, \quad \lim _{s \rightarrow \infty} \Lambda(s)=0,
$$

and

$$
\lim _{x \rightarrow \pm \infty}\left|\Lambda^{(k)}(x) \cdot x\right|<\infty, \quad k=1 \ldots 5 .
$$

Then there exists a constant $M$ such that $S$ is approximated by the formula

$$
\begin{gathered}
S=\int_{0}^{\infty}\left[A_{1}\left(\frac{-s}{\sqrt[3]{3 \omega}}\right)\left(\Lambda^{\prime}(s+\sigma)+\frac{c \mu^{2} \omega}{2} \Lambda^{\prime \prime}(s+\sigma)+\frac{c \mu^{2} s^{3}}{12} \Lambda^{\prime \prime}(s+\sigma)\right)-\right. \\
\left.-\operatorname{Ai}\left(\frac{-s}{\sqrt[3]{3 \omega}}\right) \frac{(3 \omega)^{2 / 3}}{2 s^{2}} \cdot \frac{c \mu^{2} s^{3}}{6} \Lambda^{\prime \prime}(s+\sigma)+\operatorname{Ai}^{\prime}\left(\frac{-s}{\sqrt[3]{3 \omega}}\right) \frac{(3 \omega)^{1 / 3}}{2 s} \cdot \frac{c \mu^{2} s^{3}}{6} \Lambda^{\prime \prime}(s+\sigma)\right] d s- \\
-\int_{-\infty}^{0}\left[A_{2}\left(\frac{-s}{\sqrt[3]{3 \omega}}\right)\left(\Lambda^{\prime}(s+\sigma)+\frac{c \mu^{2} \omega}{2} \Lambda^{\prime \prime}(s+\sigma)+\frac{c \mu^{2} s^{3}}{12} \Lambda^{\prime \prime}(s+\sigma)\right)+\right. \\
\left.+\operatorname{Ai}\left(\frac{-s}{\sqrt[3]{3 \omega}}\right) \frac{(3 \omega)^{2 / 3}}{2 s^{2}} \cdot \frac{c \mu^{2} s^{3}}{6} \Lambda^{\prime \prime}(s+\sigma)-\operatorname{Ai}^{\prime}\left(\frac{-s}{\sqrt[3]{3 \omega}}\right) \frac{(3 \omega)^{1 / 3}}{2 s} \cdot \frac{c \mu^{2} s^{3}}{6} \Lambda^{\prime \prime}(s+\sigma)\right] d s+\varphi(\sigma, \omega), \\
|\varphi(\sigma, \omega)| \leq M \mu^{4} \omega^{2}=M\left(\frac{t}{\rho}\right)^{2},
\end{gathered}
$$

where

$$
A_{1}(x)=\int_{-\infty}^{x} \operatorname{Ai}(z) d z, \quad A_{2}(x)=\int_{x}^{\infty} \operatorname{Ai}(z) d z
$$


P r o o f. Conditions (3.6) provide the same decaying of $R$ and its derivatives as $\sigma \rightarrow \pm \infty$. After integration of (3.5) by parts twice, using the properties of Airy functions, we have

$$
S=-\int_{0}^{\omega} \int_{-\infty}^{\infty} \frac{\sigma-\sigma^{\prime}}{\left(3\left(\omega-\omega^{\prime}\right)\right)^{4 / 3}} \operatorname{Ai}\left(\frac{\sigma-\sigma^{\prime}}{\sqrt[3]{3\left(\omega-\omega^{\prime}\right)}}\right) \frac{\partial R\left(\sigma^{\prime}, \mu^{2} \omega^{\prime}\right)}{\partial \sigma^{\prime}} d \sigma^{\prime} d \omega^{\prime} .
$$

We split the integral with respect to $\sigma^{\prime}$ from $-\infty$ to $\infty$ into two integrals (from $-\infty$ to $\sigma$ and from $\sigma$ to $\infty$ ), let us also make change of variables

$$
s=\sigma^{\prime}-\sigma, \quad z=\frac{-s}{\sqrt[3]{3\left(\omega-\omega^{\prime}\right)}},
$$

and change the order of integration. All integrals above exist due to (3.6), (3.3). We have:

$$
\begin{gathered}
S=-\int_{-\infty}^{0} \int_{\frac{-s}{\sqrt[3]{3 \omega}}}^{\infty} \operatorname{Ai}(z) \frac{\partial R\left(s+\sigma, \mu^{2} \omega^{\prime}\right)}{\partial s} d z d s+\int_{0}^{\infty} \int_{-\infty}^{\frac{-s}{3}} \operatorname{Ai}(z) \frac{\partial R\left(s+\sigma, \mu^{2} \omega^{\prime}\right)}{\partial s} d z d s \\
\omega^{\prime}=\omega+\frac{1}{3}\left(\frac{s}{z}\right)^{3} .
\end{gathered}
$$

For $\frac{\partial R}{\partial \sigma}$, using (3.3), we have:

$$
\frac{\partial R}{\partial \sigma}=\frac{1}{c \mu^{2} \omega^{\prime}} \int_{0}^{1} \frac{d \Lambda\left(\sigma+c \mu^{2} \omega^{\prime} y\right)}{d y} d y=\frac{\Lambda\left(\sigma+c \mu^{2} \omega^{\prime}\right)-\Lambda(\sigma)}{c \mu^{2} \omega^{\prime}} .
$$

For small $\mu$ we expand this expression into the Taylor series:

$$
\frac{\partial R}{\partial \sigma}=\Lambda^{\prime}(\sigma)+\frac{c \mu^{2} \omega^{\prime}}{2} \Lambda^{\prime \prime}(\sigma)+O\left(\mu^{4} \omega^{\prime 2}\right)
$$

and place it in $S$. We have:

$$
\begin{aligned}
S & =\int_{0}^{\infty} \int_{-\infty}^{\frac{-s}{\sqrt[3]{3 \omega}}} \operatorname{Ai}(z)\left(\Lambda^{\prime}(s+\sigma)+\frac{c \mu^{2} \omega^{\prime}}{2} \Lambda^{\prime \prime}(s+\sigma)+O\left(\mu^{4} \omega^{2}\right)\right) d z d s- \\
& -\int_{-\infty}^{0} \int_{\frac{-s}{\sqrt[3]{3 \omega}}}^{\infty} \operatorname{Ai}(z)\left(\Lambda^{\prime}(s+\sigma)+\frac{c \mu^{2} \omega^{\prime}}{2} \Lambda^{\prime \prime}(s+\sigma)+O\left(\mu^{4} \omega^{\prime 2}\right)\right) d z d s
\end{aligned}
$$

For $A_{1}, A_{2}$ it holds

$$
\begin{aligned}
& \int_{-\infty}^{x} \frac{\operatorname{Ai}(z)}{z^{3}} d z=-\frac{x^{-2}}{2} \operatorname{Ai}(x)-\frac{x^{-1}}{2} \operatorname{Ai}^{\prime}(x)+\frac{1}{2} A_{1}(x), \\
& \int_{x}^{\infty} \frac{\operatorname{Ai}(z)}{z^{3}} d z=\frac{x^{-2}}{2} \operatorname{Ai}(x)+\frac{x^{-1}}{2} \operatorname{Ai}^{\prime}(x)+\frac{1}{2} A_{2}(x) .
\end{aligned}
$$


So

$$
\begin{gathered}
S=\int_{0}^{\infty}\left[A_{1}\left(\frac{-s}{\sqrt[3]{3 \omega}}\right)\left(\Lambda^{\prime}(s+\sigma)+\frac{c \mu^{2} \omega}{2} \Lambda^{\prime \prime}(s+\sigma)+\frac{c \mu^{2} s^{3}}{12} \Lambda^{\prime \prime}(s+\sigma)\right)-\right. \\
\left.-\operatorname{Ai}\left(\frac{-s}{\sqrt[3]{3 \omega}}\right) \frac{(3 \omega)^{2 / 3}}{2 s^{2}} \cdot \frac{c \mu^{2} s^{3}}{6} \Lambda^{\prime \prime}(s+\sigma)+\operatorname{Ai}^{\prime}\left(\frac{-s}{\sqrt[3]{3 \omega}}\right) \frac{(3 \omega)^{1 / 3}}{2 s} \cdot \frac{c \mu^{2} s^{3}}{6} \Lambda^{\prime \prime}(s+\sigma)\right] d s+O\left(\mu^{4} \omega^{2}\right)- \\
-\int_{-\infty}^{0}\left[A_{2}\left(\frac{-s}{\sqrt[3]{3 \omega}}\right)\left(\Lambda^{\prime}(s+\sigma)+\frac{c \mu^{2} \omega}{2} \Lambda^{\prime \prime}(s+\sigma)+\frac{c \mu^{2} s^{3}}{12} \Lambda^{\prime \prime}(s+\sigma)\right)+\right. \\
\left.+\operatorname{Ai}\left(\frac{-s}{\sqrt[3]{3 \omega}}\right) \frac{(3 \omega)^{2 / 3}}{2 s^{2}} \cdot \frac{c \mu^{2} s^{3}}{6} \Lambda^{\prime \prime}(s+\sigma)-\operatorname{Ai}^{\prime}\left(\frac{-s}{\sqrt[3]{3 \omega}}\right) \frac{(3 \omega)^{1 / 3}}{2 s} \cdot \frac{c \mu^{2} s^{3}}{6} \Lambda^{\prime \prime}(s+\sigma)\right] d s+O\left(\mu^{4} \omega^{2}\right) .
\end{gathered}
$$

This formula admits formal differentiation with respect to $\sigma$ since (3.8), (3.9) give the same formulas

$$
\frac{\partial^{2} R}{\partial \sigma^{2}}=\frac{\Lambda^{\prime}\left(\sigma+c \mu^{2} \omega^{\prime}\right)-\Lambda^{\prime}(\sigma)}{c \mu^{2} \omega^{\prime}}=\Lambda^{\prime \prime}(\sigma)+\frac{c \mu^{2} \omega^{\prime}}{2} \Lambda^{\prime \prime \prime}(\sigma)+O\left(\mu^{4} \omega^{\prime 2}\right)
$$

Also

$$
\begin{gathered}
\frac{\partial^{3} R}{\partial \sigma^{3}}=\Lambda^{\prime \prime \prime}(\sigma)+\frac{c \mu^{2} \omega^{\prime}}{2} \Lambda^{(4)}(\sigma)+O\left(\mu^{4} \omega^{2}\right), \quad \frac{\partial^{4} R}{\partial \sigma^{4}}=\Lambda^{(4)}(\sigma)+\frac{c \mu^{2} \omega^{\prime}}{2} \Lambda^{(5)}(\sigma)+O\left(\mu^{4} \omega^{\prime 2}\right) . \\
\frac{d}{d \omega^{\prime}} \frac{\partial R\left(\sigma, \mu^{2} \omega^{\prime}\right)}{\partial \sigma}=\frac{c \mu^{2}}{2} \Lambda^{\prime \prime}(\sigma)+O\left(\mu^{4} \omega^{\prime}\right) .
\end{gathered}
$$

Therefore

$$
\begin{gathered}
\frac{\partial S}{\partial \omega}=\int_{-\infty}^{\infty} \operatorname{Ai}\left(\frac{-s}{\sqrt[3]{3 \omega}}\right) \Lambda^{\prime}(s+\sigma) d s-\int_{-\infty}^{0} \int_{-s}^{\infty} \operatorname{Ai}(z) H(s, z) d z d s+\int_{0}^{\infty} \int_{-\infty}^{\frac{-s}{3 \omega}} \operatorname{Ai}(z) H(s, z) d z d s . \\
H(s, z)=\frac{d}{d \omega^{\prime}} \frac{\partial R\left(s+\sigma, \mu^{2} \omega^{\prime}\right)}{\partial s} \cdot \frac{d \omega^{\prime}}{d \omega}=\frac{d}{d \omega^{\prime}} \frac{\Lambda\left(s+\sigma+c \mu^{2} \omega^{\prime}\right)-\Lambda(s+\sigma)}{c \mu^{2} \omega^{\prime}}
\end{gathered}
$$

that proves Proposition 1.

For the approximate solution $\tilde{u}=R+S$ we denote

$$
\begin{gathered}
\mathcal{K} \tilde{u}=\frac{\partial \tilde{u}}{\partial \omega}+\mu^{2} \tilde{u} \frac{\partial \tilde{u}}{\partial \sigma}+\frac{\partial^{3} \tilde{u}}{\partial \sigma^{3}}, \quad m_{1}(\sigma, \omega)=|\mathcal{K} \tilde{u}|, \\
m_{2}(\sigma, \omega)=\left|\frac{\partial \tilde{u}}{\partial \omega}\right|+\left|\mu^{2} \tilde{u} \frac{\partial \tilde{u}}{\partial \sigma}\right|+\left|\frac{\partial^{3} \tilde{u}}{\partial \sigma^{3}}\right|, \quad A_{\mu}(\omega)=\frac{\left\|m_{1}(\cdot, \omega)\right\|_{L_{1}}}{\left\|m_{2}(\cdot, \omega)\right\|_{L_{1}}}
\end{gathered}
$$

Due to the equation (3.4) we have $\mathcal{K} \tilde{u}=\frac{\partial R}{\partial \omega}+\mu^{2} \tilde{u} \frac{\partial \tilde{u}}{\partial \sigma}$. We say that $\tilde{u}$ is an $L_{1}$-asymptotic solution, if the function $A_{\mu}(\omega)$ is uniformly small for $\omega \in\left(0, \omega_{1}\right)$.

Exactly this ratio is important, but not the value of $\mathcal{K} \tilde{u}$ by itself, because generally speaking, it can be great. Note that the used notion of $L_{1}$-asymptotic solution differs from the standard definition of the formal asymptotic solution in form of infinite number of terms, giving arbitrarily small error by substituting it into the equation. 
In the case where the asymptotic solution is constructed in the form of a function (not a series), even the smallness of the remainders (after the substitution of this function into the equation) by itself cannot serve as a criterion of its suitability, since derivatives can be of the same order of smallness; hence, the value of the remainders should be compared with something. It is naturally to compare with absolute values of individual terms, included in the equation.

\section{Numerical computations}

In this section there are graphs of the $L_{1}$-asymptotic solutions of the problem. The system Matlab R2012a on the "Uran" supercomputer of IMM UB RAS was used for computations.

The double integration of (3.7) and approximate single integration (3.10) were performed. We used the method of trapezes with the integration step 0.1 .

1. Initial function was chosen in the form $\Lambda=\frac{c}{6 \pi}\left(\operatorname{arctg} x-\frac{\pi}{2}\right)$. In this case

$$
R=-\frac{c}{12}+\frac{\rho}{6 \pi t}\left[\left(\sigma+\frac{c t}{\rho}\right) \operatorname{arctg}\left(\sigma+\frac{c t}{\rho}\right)-\sigma \operatorname{arctg} \sigma-\frac{1}{2} \ln \left(1+\frac{2 c t \rho \sigma+c^{2} t^{2}}{\rho^{2}\left(1+\sigma^{2}\right)}\right)\right]
$$

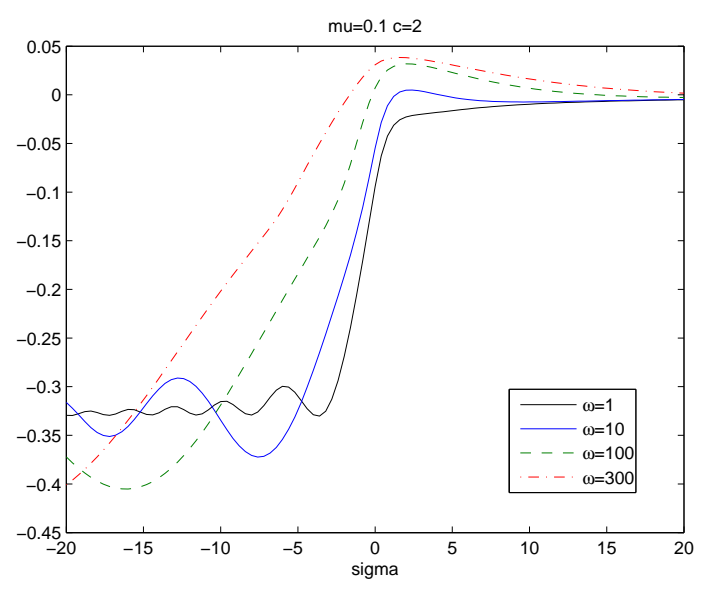

Figure 1. Function $\tilde{u}$ for different values of $\omega$

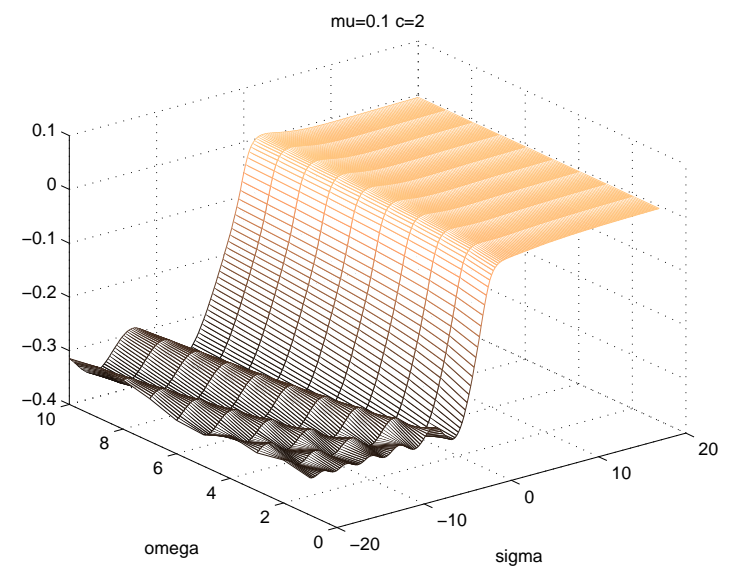

Figure 2. Function $\tilde{u}$ for $\omega \in(1,10), \quad \sigma \in(-20,20)$.

2. Numerical estimation of the KdV operator. The functions $m 1, m 2$ and $A_{\mu}(\omega)$ are introduced in $(3.11)$.

As we can see in Fig. 3, 4, the value of $A_{\mu}(\omega)$ is of the order of 0.01 for $\mu=0.1$, and of the order of 0.0001 in the case $\mu=0.01$. Numerical computations for smaller $\mu$ show that $A_{\mu}$ has the order $\mu^{2} \omega$. We emphasize that it is important for the value of $A_{\mu}(\omega)$ to be small, and the value $m_{1}$ does not necessary have to be small. Thus, according to (3.2), the obtained asymptotic solution describes only the initial stage of the propagation of the rarefaction wave for $t \ll \rho$ and further investigation is needed. 


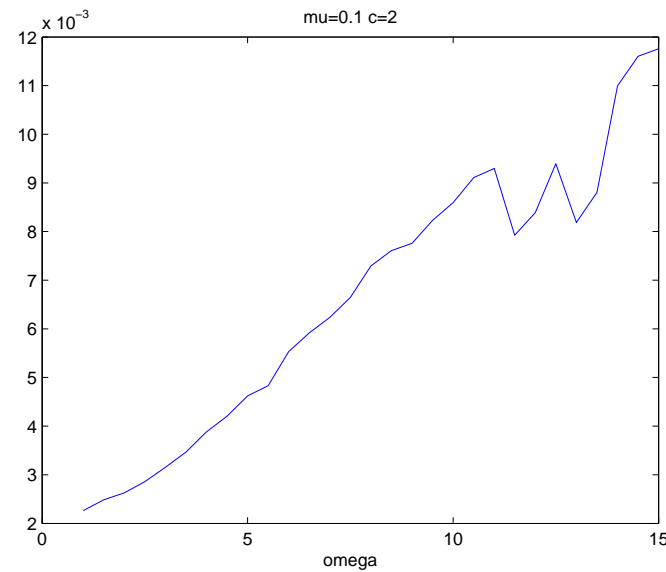

Figure 3. $A_{\mu}(\omega)$ for $\mu=0.1$.

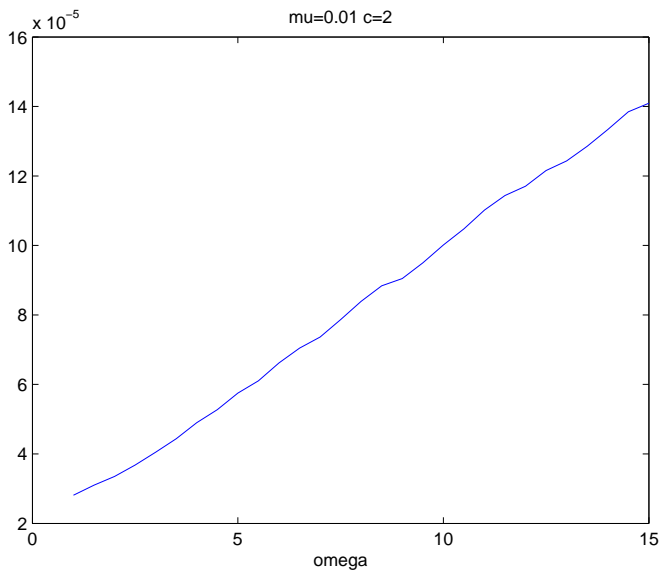

Figure 4. $A_{\mu}(\omega)$ for $\mu=0.01$.

3. In this section we compare two ways of integration. While obtaining of $S$ we may use one of two choices: the formulas (3.7), (3.8), and the formula (3.10) after approximation (3.9). This approximation helps us to simplify the computing due to removing the integration with respect to $z$, and the integral (3.10) with respect to $s$ only remains. It reduces the duration of the computations up to 30 times.

We denote (3.7) as $f_{1-}+f_{1+}$ and (3.10) as $f_{2-}+f_{2+}$ and compare them. We have

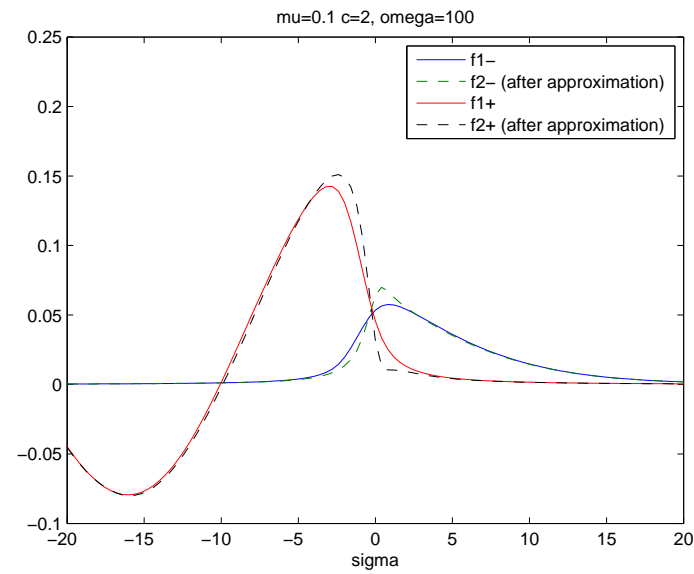

Figure 5. Functions $f_{1 \pm}, f_{2 \pm}$ for $\omega=100$.

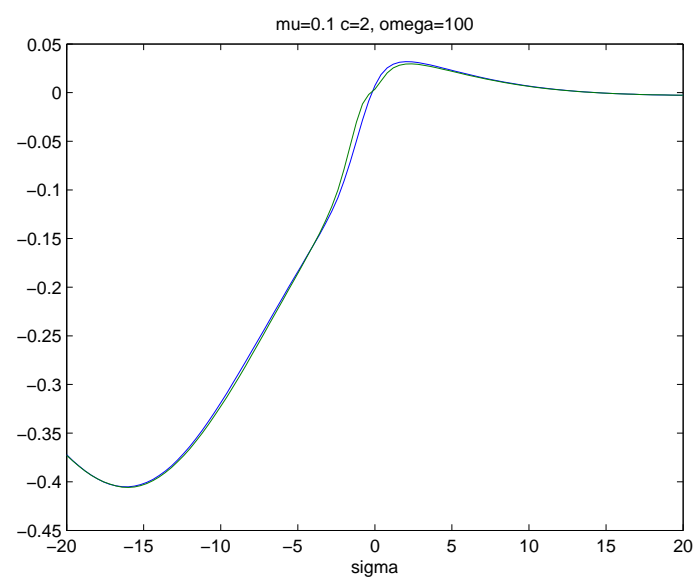

Figure 6. Functions $\tilde{u}=R+S$ for $\omega=100$ before and after approximation (3.10).

We see that although $\mu^{2} \omega=1$ for $\mu=0.1, \omega=100$, we get satisfactory result for applying the approximation (3.10).

The following graph shows that for $\omega=10$ the functions $f_{1 \pm}$ almost match $f_{2 \pm}$. 


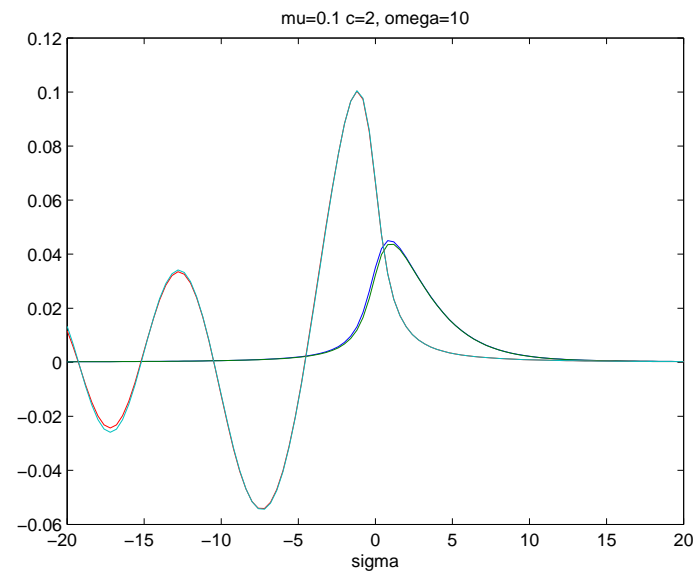

Figure 7. Functions $f_{1 \pm}, f_{2 \pm}$ for $\omega=10$.

4. Consider non-monotonic case with the initial function in the form

$$
\Lambda=\frac{c}{6 \pi}\left(\operatorname{arctg} x-\frac{\pi}{2}+\frac{5}{1+x^{2}}\right) .
$$

We get

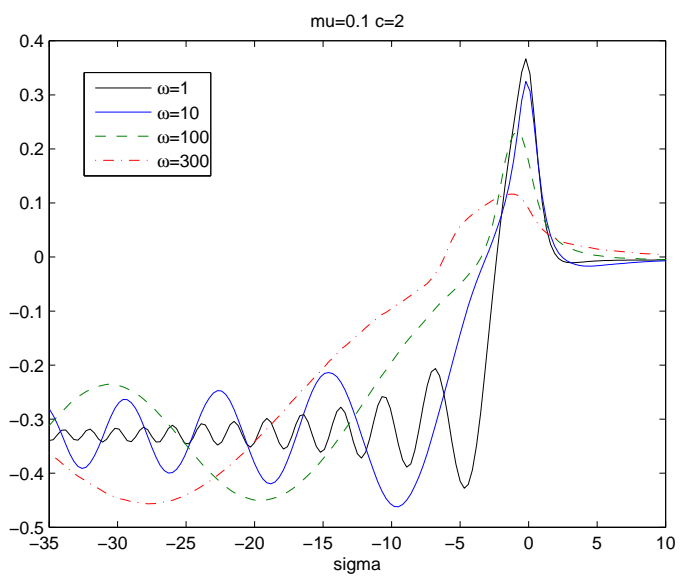

Figure 8. Function $\tilde{u}$ for different values of $\omega$ in nonmonotonic case.

\section{REFERENCES}

1. Gurevich A.V., Pitaevskii L.P. Nonstationary structure of a collisionless shock wave // Sov. Phys.JETP, 1974. Vol. 38, no. 2. P. 291-297.

2. Gurevich A.V., Krylov A.L., El' G.A. Breaking of a Riemann wave in dispersive hydrodynamics // JETP Lett., 1991. Vol. 54, no. 102. P. 102-107.

3. Krylov A.L., Khodorovskii V.V., El' G.A. Evolution of a nonmonotonic perturbation in Kortewegde Vries hydrodynamics // JETP Lett., 1992. Vol. 56, no. 6. P. 323-327.

4. Mazur N.G. Quasiclassical asymptotics of the inverse scattering solutions of the KdV equation and the solution of Whitham's modulation equations // Theoret. and Math. Phys. 1996. Vol. 106, no. 1. P. 35-49. DOI: 10.1007/BF02070761

5. Khruslov E.Ya. Asymptotics of the solutions of the Cauchy problem for the Korteweg-de Vries equation with initial data of step type // Math. USSR-Sb., 1976. Vol. 28, no. 2 pp. 229-248. 
6. Cohen A. Solutions of the Korteweg-de Vries equation with steplike initial profile // Comm. Partial Diff. Eq., 1984. Vol. 9, no. 8. P. 751-806.

7. S. Venakides Long time asymptotics of the Korteweg-de Vries equation // Transactions of AMS, 1986. Vol. 293, no. 1. P. 411-419.

8. Suleimanov B.I. Solution of the Korteweg-de Vries equation which arises near the breaking point in problems with a slight dispersion. // JETP Lett. 1993. Vol. 58, no. 11. P. 849-854.

9. Suleimanov B.I. Asymptotics of the Gurevich-Pitaevskii universal special solution of the Korteweg-de Vries equation as $|x| \rightarrow \infty / /$ Proc. Steklov Inst. Math. (Suppl.), 2013. Vol. 281. Suppl. 1. P. $137-145$. doi: $10.1134 /$ S0081543813050131

10. Kappeler T. Solutions of the Korteweg-de Vries equation with steplike initial data // J. Diff. Eq.,1986. Vol. 63, no. 3. P. 306-331.

11. Bondareva I.N. The Korteweg-de Vries equation in classes of increasing functions with prescribed asymptotics as $|x| \rightarrow \infty / /$ Math. USSR-Sb. 1985. Vol. 50, no. 1. P. 125-135. DOI: 10.1070/SM1985v050n01ABEH002736

12. Zakharov S.V. Renormalization in the Cauchy problem for the Korteweg-de Vries equation // Theoret. and Math. Phys., 2013. Vol. 175, no. 2. P. 592-595. DOI: 10.1007/s11232-013-0048-7

13. Teodorovich E.V. Renormalization group method in the problems of mechanics // J. Appl. Math. Mech., 2004. Vol. 68, no. 2. P. 299-326.

14. Il'in A.M. Matching of asymptotic expansions of solutions of boundary value problems. Editor - AMS. 1992. 281 p. ISBN: 978-0-8218-4561-5.

15. Zakharov S.V. The Cauchy problem for a quasilinear parabolic equation with two small parameters // Dokl. Math., 2008. Vol. 78, no. 2. P. 769-770.

16. Zakharov S.V. The Cauchy problem for a quasilinear parabolic equation with a large initial gradient and low viscosity. // Comput. Math. Math. Phys., 2010. Vol. 50, no.4. P. 665-672. DOI: 10.1134/S0965542510040081

17. Egorova I., Gladka Z., Lange T.L., Teschl G. On the inverse scattering transform method for the Korteweg-de Vries equation with steplike initial data. University of Vienna. Prepr. Wien, 2014.

18. Egorova I., Grunert K., Teschl G. On the Cauchy problem for the Korteweg-de Vries equation with steplike finite-gap initial data I. Schwartz-type perturbations // Nonlinearity, 2009. Vol. 22. P. 14311457.

19. Egorova I., Teschl G. On the Cauchy problem for the Korteweg-de Vries equation with steplike finite-gap initial data II. Perturbations with finite moments // J. d'Analyse Math., 2011. Vol. 115, no. 1. P. 71-101.

20. Grunert K. Teschl G. Long-time asymptotics for the Korteweg-de Vries equation via nonlinear steepest descent // Math. Phys. Anal. Geom., 2009. Vol. 12, no. 3. P. 287-324. DOI: 10.1007/s11040-0099062-2

21. Kotlyarov V.P., Minakov A.M. Riemann-Hilbert problem to the modified Korteveg-de Vries equation: Long-time dynamics of the step-like initial data // J. Math. Phys., 2010. Vol. 51, no. 9, 093506. DOI: $10.1063 / 1.3470505$

22. Leach J.A., Needham D.J. The large-time development of the solution to an initial- value problem for the Korteweg-de Vries equation: I. Initial data has a discontinuous expansive step // Nonlinearity. 2008. Vol. 21, no. 10. P. 2391-2408. DOI: 10.1088/0951-7715/21/10/010

23. Novokshenov V.Yu. Time asymptotics for soliton equations in problems with step initial conditions // J. Math. Sci. (N.Y.), 2005. Vol. 125, no. 5. P. 717-749.

24. Baranetskii V.B., Kotlyarov V.P. Asymptotic behavior in the trailing edge domain of the solution of the KdV equation with an initial condition of the "threshold type" // Theoret. and Math. Phys., 2001. Vol. 126, no. 2. P. 175-186. DOI: 10.1023/A:1005291626477

25. Brekhovskikh V.V., Gorev V.V. Collisionless damping of soliton solutions of Korteweg - de Vries equation, the modified Korteweg - de Vries equation and nonlinear Schrödinger equation // Izvestiya vuzov. Povolzhskiy region. Physical-mathematical sciences, 2015. No. 2. P. 190-202. [in Russian]

26. Gladka Z.N. On solutions of the Korteweg-de Vries equation with initial data of step-type // Dop. National Academy of Sciences of Ukraine. 2015. Vol. 2. [in Russian]

27. Gladka Z.N. On the reflection coefficient of the Schrödinger operator with a smooth potential // Dop. National Academy of Sciences of Ukraine. 2014. Vol. 9. [in Russian] 
28. Berezin Yu.A., Karpman V.I. Nonlinear evolution of disturbances in plasmas and other dispersive media // JETP., 1967. Vol. 24, no. 5. P. 1049-1056.

29. Fogaca D.A., Navarra F.S., Ferreira Filho L.G. KdV solitons in a cold quark gluon plasma // Physical Review D., 2011. 84, 054011. DOI: 10.1103/PhysRevD.84.054011

30. Frank Verheest, Carel P. Olivier, Willy A. Hereman. Modified Korteweg-de Vries solitons at supercritical densities in two-electron temperature plasmas // J. of Plasma Physics, 2016. Vol. 82, art. no. 905820208,13 p.

31. Misra A.P., Barman Arnab. Landau damping of Gardner solitons in a dusty bi-ion plasma // Phys. Plasmas, 2015. Vol. 22, 073708.

32. Dutykh D., Tobisch E. Observation of the Inverse Energy Cascade in the modified Korteweg-de Vries Equation. arXiv:1406.3784

33. Zakharov S.V., Elbert A.E. Modelling compression waves with a large initial gradient in the Korteweg-de Vries hydrodynamics // Ufa Math. J., 2017. Vol. 9, no. 1. P. 41-53.

34. Ablowitz M.J., Baldwin D.E., Hoefer M.A. Soliton generation and multiple phases in dispersive shock and rarefaction wave interaction// Physical Review E, 2009. Vol. 80, 016603.

35. Kyrylo Andreiev, Iryna Egorova, Till Luc Lange, Gerald Teschl. Rarefaction waves of the Korteweg-de Vries equation via nonlinear steepest descent. arXiv:1602.02427 